\title{
The Software Design of Natural Rubber Plantation Temperature and Hu- midity Monitoring System Based on ZigBee
}

\author{
Jin Zhi-yang ${ }^{1,2}$, Li Zhong-xing ${ }^{1, *}$, Jiang Hong ${ }^{3}$, Sun Li-qin ${ }^{1}$ and Xiao Wen ${ }^{1}$ \\ ${ }^{1}$ College of Automotive and Traffic Engineering of Jiangsu University, Zhenjiang, Jiangsu, China \\ ${ }^{2}$ College of Electrical and Mechanical Engineering of Hainan University, Haikou, Hainan, China \\ ${ }^{3}$ College of Mechanical Engineering of Jiangsu University, Zhenjiang, Jiangsu, China
}

\begin{abstract}
The temperature and humidity of the climate affect forest of natural rubber latex yield enormously. Peasants need to control the temperature and humidity manually based on the weather forecast after growing the trees. It costs enormous human resources. Meanwhile the diseases caused by an unsuitable temperature or humidity cannot be foreseen. Aiming at monitoring the temperature, humidity and illumination intensity in the growing environment of a tropical crop--rubber tree, this article builds ZigBee wireless sensor network, and designs the software structure of this system based on fulfilling the hardware designing requirements. Combining Cluster tree algorithm in ZigBee network with AODVjr algorithm, this article enhances the utility of ZigBee wireless sensor network efficiently, and proves the effectiveness of ZigBee wireless sensor network technique in the tropical crops monitoring system. In order to realize the modern forestry management, the distribution of the temperature and humidity in the whole woods should be available to collect to minutely control the irrigation systems, insulation systems, etc. and then providing the best surroundings to guarantee a high quality.
\end{abstract}

Keywords: ZigBee Technique, Software design, Rubber plantation, Temperature and humidity collecting, Algorithm.

\section{INTRODUCTION}

The rubber tree is a plant of large tree, native to Brazil. In the early $20^{\text {th }}$ century, rubber tree was introduced successfully to China. Rubber tree needs high temperature, high humidity, calm winds and fertile soil, with the temperature $26 \sim 27^{\circ} \mathrm{C}$. It grows and produces rubber at temperature $20 \sim 30^{\circ} \mathrm{C}$, but gets destroyed at temperature below $5^{\circ} \mathrm{C}$. Its requirement of average annual precipitation is 1150 to 2500 $\mathrm{mm}$, but it should not be planted in a low place.

Temperature directly affects the growth, development, rubber production and even survival of the rubber tree. It is a major factor to restrict the geographical distribution of the rubber tree.

\subsection{The Temperature Indicators of Rubber Tree's Growth and Development}

Measured by average temperature: When the temperature is $10^{\circ} \mathrm{C}$, cells undergo mitosis, which is good for the growth and production of rubber in $20^{\circ} \mathrm{C} \sim 30^{\circ} \mathrm{C}$; the critical temperature for tissue differentiation is $15^{\circ} \mathrm{C}$, while $18{ }^{\circ} \mathrm{C}$ is critical temperature for normal growth; it is good to produce rubber in $20^{\circ} \mathrm{C} \sim 30^{\circ} \mathrm{C}$; rubber trees grow most vigorously in $26^{\circ} \mathrm{C} \sim 27^{\circ} \mathrm{C}$

*Address correspondence to this author at the College of Automotive and Traffic Engineering of Jiangsu University, Zhenjiang, Jiangsu, China;

E-mail: jinzhiyang94@163.com

\subsection{The Temperature Indicators of Rubber Tree's Pho- tosynthesis}

Measured by actual temperature: When the temperature is below $10^{\circ} \mathrm{C}$, it has harmful effects on the metabolism of seedlings and the photosynthesis is stopped; $5^{\circ} \mathrm{C} \sim 30^{\circ} \mathrm{C}$ is the optimum temperature for photosynthesis; rubber tree's respiration exceeds photosynthesis. Its growth is inhibited, with leaves burnt.

\subsection{The Temperature Indicators of Rubber Tree's Latex Synthesis}

Measured by average temperature: Rubber tree's Latex synthesis works at $18^{\circ} \mathrm{C} \sim 28^{\circ} \mathrm{C}$ and best at $22^{\circ} \mathrm{C} \sim 25^{\circ} \mathrm{C}$.

\subsection{The Optimal Conditions of Producing Rubber}

The tree produces best at temperatures $19^{\circ} \mathrm{C} \sim 24^{\circ} \mathrm{C}$ with a relative humidity greater than $80 \%$.

\subsection{Harmful Temperature to the Rubber Tree}

When the forest temperature is $<5^{\circ} \mathrm{C}$, rubber tree gets affected from different degrees of chilling injuries, such as RRIM600 in which plants produce a small amount of gum and suffer from explosion of skin; When the forest temperature is $<0^{\circ} \mathrm{C}$, treetops and tree trunks would be withered and below $<-2^{\circ} \mathrm{C}$ the roots explosion skin plastic flow phenomenon appears and the rubber tree gets serious chilling injuries. When the actual temperature is $>40^{\circ} \mathrm{C}$, in addition to causing respiration increased and invalid consumption, being not conducive to the gum production, it also directly destructs 
gum trees, like sprout leaves burnt, seedlings withered necrosis, saplings bark burnt, forcing fallen leaves.

In summary of those temperature indicators, rubber tree's fast-growing, high-yield, photosynthetic optimum temperature range is $18^{\circ} \mathrm{C} \sim 28^{\circ} \mathrm{C}$. The more accumulated temperature it gets in an optimum temperature, the longer growing season and tapping period will it get.

All the physiological activities of the rubber tree need water. Photosynthesis, transpiration needs water, and transport of organic and inorganic nutrients, synthesis and decomposition of organic matter need water too.

Suitable precipitation index for the growth of rubber trees and rubber producing is the annual rainfall of $1,500 \mathrm{~mm}$ or more. Annual rainfall from 1500 to $2500 \mathrm{~mm}$, relative humidity of $80 \%$ or more and the annual rainy days $>150$ days are best suited to the growth and production of rubber. When the rainfall is greater than $2500 \mathrm{~mm}$ and precipitation days are excessive, it is not conducive to tapping rubber, and diseases are of high epidemicity. If the water condition is measured by monthly rainfall, when monthly rainfall is $>100 \mathrm{~mm}$, the monthly rain days are $>10$ days, it is suitable for rubber tree's growth; it will be best for the rubber tree's growth when the monthly rainfall is $>150 \mathrm{~mm}[1,2]$.

Knowing the demands of rubber trees, peasants need to control the temperature and humidity manually based on the weather forecast after growing the trees. It cost enormous human resources. Meanwhile the diseases caused by a wrong temperature or humidity cannot be foreseen. In order to realize the modern forestry management, the distribution of the temperature and humidity in the whole woods should be available to collect to minutely control the irrigation systems, insulation systems, etc., then providing the best surroundings to guarantee a high quality, liberating the human resources, reducing the peasants' burdens and the improving the progress of modernized technology. The occurring of the wireless sensor network based on Zigbee raised an efficient and scientific solution to collect the temperature and humidity of the rubber woods and provided a theoretical basis to realize the modern forestry management.

\section{BRIEF INTRODUCTION OF ZIGBEE}

ZigBee technology is a short-range, low-complexity, low-power, low-rate, low-cost two-way wireless communication technology. It is mainly used for short distance, low power consumption and low transmission rate between the various electronic devices for data transmission and the typical application of periodic data, intermittent data, and data transmission of the low reaction time. ZigBee module is an Internet of things wireless data terminal; it utilizes the ZigBee network to provide users with wireless data transmission capabilities. ZigBee technique is built on IEEE802.15.4 Standard. In technical protocol, physical layer and data link layer use IEEE802.15.4 Standard and ZigBee Union makes protocols of network layer and application layer. IEEE802.15.4 only standardizes the physical layer (PHY) and media access control (MAC), but the complete ZigBee protocol stack [3] is made up of senior application specification, application convergence layer, network layer, data link layer and physical layer. Network layer is based on Ad Hoc technique routing protocol and provides three kinds of network topology (star topology, tree topology, mesh topology), and it has self-organization and self-maintenance functions so that multiple devices can join ZigBee network conveniently. Application layer provides multiple application interfaces for users and users can formulate corresponding application software as required. Compared to other wireless network interconnection technique, ZigBee technique has advantages below [4]:

Low power consumption: The transmission power of the Zigbee module is only $1 \mathrm{mw}$. Mere two AA batteries can support a Zigbee facility to work for a half to two years. This is unmatched by other wireless devices.

Low overhead: The initial cost of ZigBee module is about $\$ 6$, and would be lower as $\$ 1.5 \sim 2.5$. Low cost is a key factor for ZigBee.

Low delay: Zigbee has a short response time. It can start to work from the standby within $15 \mathrm{~ms}$ and connects to the network in $30 \mathrm{~ms}$. Thus ZigBee technology is applied to the demanding delay.

Big network capacity. A Zigbee network contains 255 nodes, which can be extended to 65535 . The network topologies include three types: star topology, mesh topology and mixed type, which are convenient to join, manage and set networks.

Reliability: It adopts a collision avoidance strategy, as well as sets aside a dedicated time slot that requires a fixed bandwidth communications business, avoiding sending data competition and conflict.

Safety: Zigbee provides a function to check data integrity based on cyclic redundancy check (CRC). Authentication and certification are accessible as it uses AES-128 encryption algorithm and security mechanisms are ready for the applications.

A typical wireless sensor network is made up of three kinds of nodes: normal nodes, sink nodes and gateway nodes. Normal and sink nodes aim to collect data and realize multihop relay transmission. Gateway nodes are used to realize the transformation between current and wired signals to join the Internet and LAN. In the ZigBee network, RFD completes normal nodes while sink and gateway nodes are finished by FFD. Generally, gateway nodes, sink nodes and normal nodes are separately delivered to coordinator, router and terminal.

Coordinator: Coordinator, also called as Gateway, is responsible for starting the whole network. It is also the first device of the network. Coordinator chooses a channel, and a network ID (also called the PAN ID, i.e. a Personal Area Network ID), and subsequently starts the entire network. The coordinator also can be used to assist in the establishment of security in the network layer and application layer binding.

N.B: Coordinator's role is mainly involved in the start-up and configuration of network. Once these are completed, the coordinator of the work like a router (or disappear). Due to the distribution characteristics of the ZigBee network itself, the entire following operation of the network is no longer dependent on the coordination existence. 


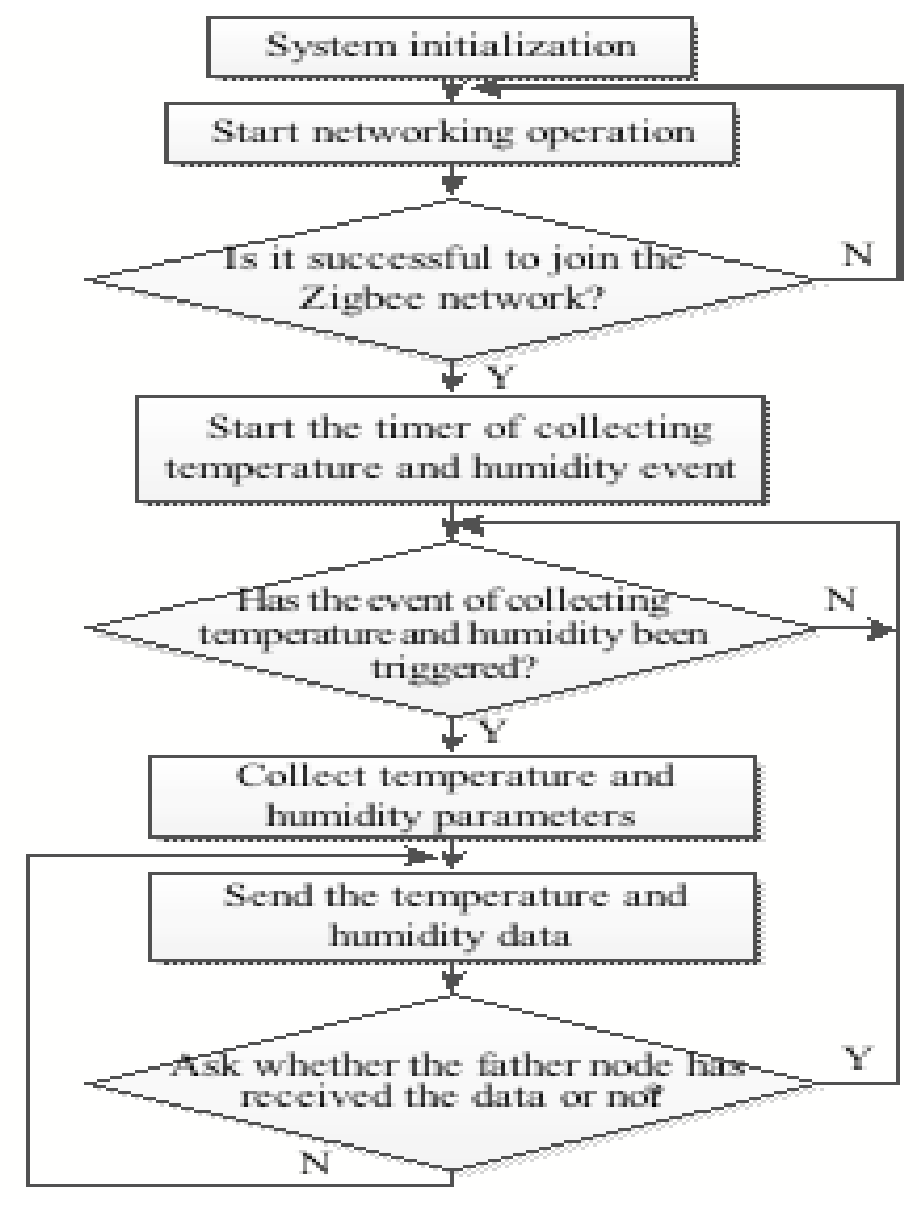

Fig. (1). Sensor nodes flowchart.

Router: Router is also called repeater. The main function of the router: is to allow other devices to join the network, multi-hop routing, and to assist in the communication of its own sub-terminal of the battery-powered devices. Typically, the router has been in active, so it must use the main power supply. However, when using such a network mode of tree mass, it allows the router to work cyclically thus batteries can be used to supply the power.

Terminal Device (End Device): The terminal device is not the responsibility of the specific maintenance of the network structure. It can be waken or just stand by, so that it can be a battery-powered device. Typically, the terminal device requires relatively small storage space (specially RAM), so it is the cheapest device in the entire network [5].

ZigBee network can contain 65535 wireless data transmission nodes at most, and the communication distance between two nodes can be 75 meters to several kilometers. In the area covered by wireless network, data can be communicated between every two nodes and transmitted to control center finally. The whole ZigBee network can also connect to other networks such as WLAN, GSM or Internet, and then remote monitoring the agriculture, environmental information is available.

The problems that need to be resolved in the software part of the Tropical Crops Monitoring System Based On ZigBee Wireless Sensor Network include: temperature and humidity information collecting, ZigBee protocol stack, communication protocol between wireless sensor module and router, communication protocol between router and coordinator, low power sleep mode. The design of software is based on Z-STACK protocol stack and IAR integrated development environment that match with $\mathrm{CC} 2430$ chip produced by TI Company [6].

\section{DESIGN OF SENSOR NODE}

This system uses the development protocol stack provided by TI Corporation, and it can achieve communication function through designing and changing the corresponding application codes.

User defines the IEEE 64 bit address of ZigBee's node. After every terminal node is switched on, ZigBee uses distributed addressing scheme to distribute 16 bit network address, it can make sure that all distributed addresses are unique, and the own distribution character of this addressing algorithm ensures that the equipment only can accept one network address with its elder communication equipment and it will contribute to the network's scalability.

When route node joins the network successfully, it will turn into dormant state. When it receives data packages transmitted from sensor node, it will be awaken and will start sending data packages into coordinator node, it will follow the wake mode: Sleep-Be awakens at the time of receiving data packages-Send data-Sleep. The software design processes are shown as Fig. (1). 
The system uses TI's development protocol stack designed to modify the application code to achieve the communication function. A wireless temperature measurement sample project based on TI is given to the preparation of the project for the system. Example is composed of LocDongle (node program) and RefNode (Temperature Measurement Node program). LocDongle sub-project's code is virtually unchanged, so if we want to add new applications, we should add wireless sensor network code in the sub-project group under RefNode APP sub layer.

Found a new main.c file and add it to the project subdirectory, given as below:

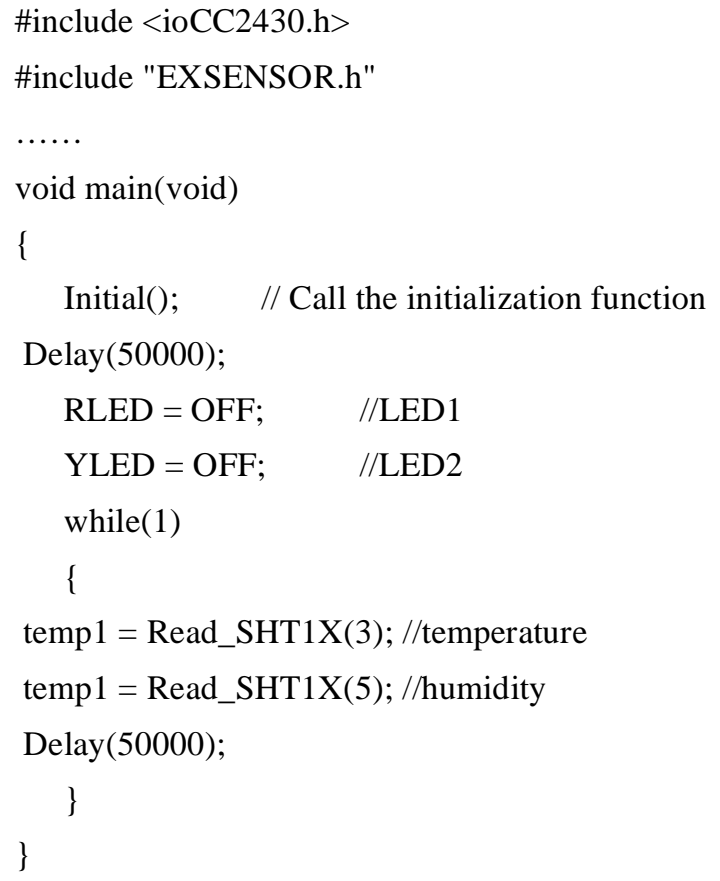

It relates to the sensor and motherboard LED lights call. The sensor drive exsensor.c is provided by the sensor chip provider. It mainly includes the A / D converter, the enabled and dormant sensor and chip data conversion. Then we add the sensor code execution exsensor.h in RefNode subprojects, given as below:

\section{\#ifndef __EXSENSOR_H}

Table1. 18 -byte Data Frame Format.
\#define_EXSENSOR_H

\#include <ioCC2430.h>

\#include "hal_types.h"

\#include "hal_defs.h"

\#include "sensor.h"

\#define SHT1X_DAT P0_5

\#define SHT1X_DAT_DIR P0DIR

\#define SHT1X_DAT_BV BV(5)

\#define SHT1X_SCK P0_4

\#define SHT1X_SCK_DIR P0DIR

\#define SHT1X_SCK_BV BV(4)

\#define SHT1X_DATA_IN st(SHT1X_DAT_DIR \&= $\sim($ SHT1X_DAT_BV);)

\#define SHT1X_DATA_OUT st(SHT1X_DAT_DIR |= SHT1X_DAT_BV;)

extern void SHT1X_PORT_INT(void);

extern uint8 WaitForSHT1XAck(void);

extern void SHT1X_REST(void);

extern void SHT1X_STAT(void);

extern uint8 SendToSHT1X(uint8 data);

extern void WaitForAminit(uint16 delay);

extern void SHT1X_INT(void);

extern uint16 Read_SHT1X(uint8 option);

\#endif

In order to make the data transmission between the Coordinator CC2430, C8051F320, PC host computer and PC remote normal, we must adopt a unified data transmission format. The present system provides the 8-byte data frame format. This format is used in the design of the entire system program thus coordinator USB interface as well as expanding available GSM interface can identify the flow of data commands. When transmitting data, the data frame format is defined as below at Table 1:

\begin{tabular}{|c|c|c|c|c|c|c|c|c|}
\hline State & Byte1 & Byte2 & Byte 3 & Byte 4 & Byte 5 & Byte 6 & Byte 7 & Byte 8 \\
\hline $\begin{array}{c}\text { Network status } \\
\text { query }\end{array}$ & $0 x F 1$ & -- & -- & -- & -- & -- & -- & -- \\
\hline Sensor status query & $0 x F 2$ & $00 / 01$ & Byte Number & -- & -- & -- & -- & -- \\
\hline $\begin{array}{l}\text { Formation of net- } \\
\text { works }\end{array}$ & $0 x F 3$ & -- & -- & -- & -- & -- & -- & -- \\
\hline Offload network & 0xF4 & -- & -- & -- & -- & -- & -- & -- \\
\hline Node query & 0xF5 & -- & -- & -- & -- & -- & -- & -- \\
\hline Sensor On & 0xF6 & $00 / 01$ & any & -- & -- & -- & -- & -- \\
\hline
\end{tabular}


Table 1. contd....

\begin{tabular}{|c|c|c|c|c|c|c|c|c|}
\hline State & Byte1 & Byte2 & Byte 3 & Byte 4 & Byte 5 & Byte 6 & Byte 7 & Byte 8 \\
\hline Sensor Off & $0 x F 7$ & $00 / 01$ & any & -- & -- & -- & -- & -- \\
\hline Node data query & $0 x F 8$ & $00 / 01$ & Byte Number & -- & -- & -- & -- & -- \\
\hline $\begin{array}{l}\text { Network Status } \\
\text { response }\end{array}$ & 0XF9 & $00 / 01$ & -- & -- & -- & -- & -- & -- \\
\hline $\begin{array}{l}\text { Sensor state re- } \\
\text { sponse }\end{array}$ & 0xFA & $00 / 01$ & Byte Number & -- & -- & -- & -- & -- \\
\hline Node information & $0 x F B$ & $\begin{array}{l}\text { Number of } \\
\text { valid bytes }\end{array}$ & -- & -- & -- & -- & -- & -- \\
\hline
\end{tabular}

\section{DESIGN OF COORDINATOR NODE}

CC2430 has four operating modes: PM0, PM1, PM2 and PM3. Because wireless sensor network node works in an unmanned backdrop, PM3 mode via external interrupt or reset to re-data transceiver is not feasible. In this design, we select the PM2 mode with higher dormant mode power consumption as compared to the PM3.

The sensor data acquisition and the terminal node dormant of the node are combined and after setting nodes in sensor data collection and successfully sending to the parent node, the node goes into sleep mode. When time is up, the next round of data collection and transmission period is started. The routing nodes go into hibernation after entering the network. Node is awakened on receiving data packet from the sensor node, and the packet is sent to the coordinator node. It is a Sleep - awakened when receiving the data packet - Transmit Data - sleep mode.

Coordinator node needs to be initialized firstly after loading power, including CC2430 chip initialization, protocol stack initialization, USAR serial initialization and hardware initialization [3]. Among those, CC2430, protocol stack and USAR serial can be initialized respectively by initialization functions what protocol stack owns, and hardware initialization function should be cut according to hardware designing of one's own. The whole protocol stack runs through an OS (operating system), so it enters the system through "osal_start_system()" function after finishing initialization. This function is also the main cyclical function in task system, and it will check all the task events and call task event handling functions for the task, which involves the event. Every task event should have an ID, and it will set the corresponding ID number as ZDO_NET_WORK_INIT [7] when some event happens, and then the network will be initialized. The flow chart of processing program on network layers is given as Fig. (2).

\section{THE DESIGN OF ROUTING NODE}

In ZigBee, "Cluster Tree \& AODVjr" routing algorithm is often used now, and nodes in network are divided into four categories named Coordinator, RN+, RN- and RFD.

Cluster Tree is a cluster tree network topology generated through the unfolding of main coordinator, and it does not have to store routing list. Most devices in the tree are FFD [8], which have retransmission function. The disadvantage of this algorithm is that the no-adaptive algorithm decides that the router it chooses is not the best one, and routing nodes close to tree root need to transmit much data, so they must store enough power.

And based on the step-by-step routing, serial number and regular broadcast mechanism of DSDV protocol, AODVjr (ad-hoc on-demand distance vector) [9] protocol enters the on-demand router finding and maintenance mechanism of DSR. AODVjr saves routing request and reply result in every non-leaf node implicitly, and limits the scope to search and find destination node by expanding ring research. Compared to DSR, the advantage of AODVjr is that not every data package needs to contain source router, and it lowers the routing protocol cost. AODVjr is a pure on-demand routing protocol, and those nodes not in path do not save routing information and do not join into the exchange of routing list.

According to the analysis to Cluster routing algorithmic and AODVjr routing algorithmic, we combine them together in our ZigBee network, and build Mesh topology structure network through mixture routing way, which combines AODVjr and scalar tree routing. According to the analysis to Tree routing and AODVjr on the above, we put forward routing way based on data character. It means that in the mesh network, which consists of two kinds of routing algorithm, according to the different data transmitting character, we choose different routing ways by setting the Discover 


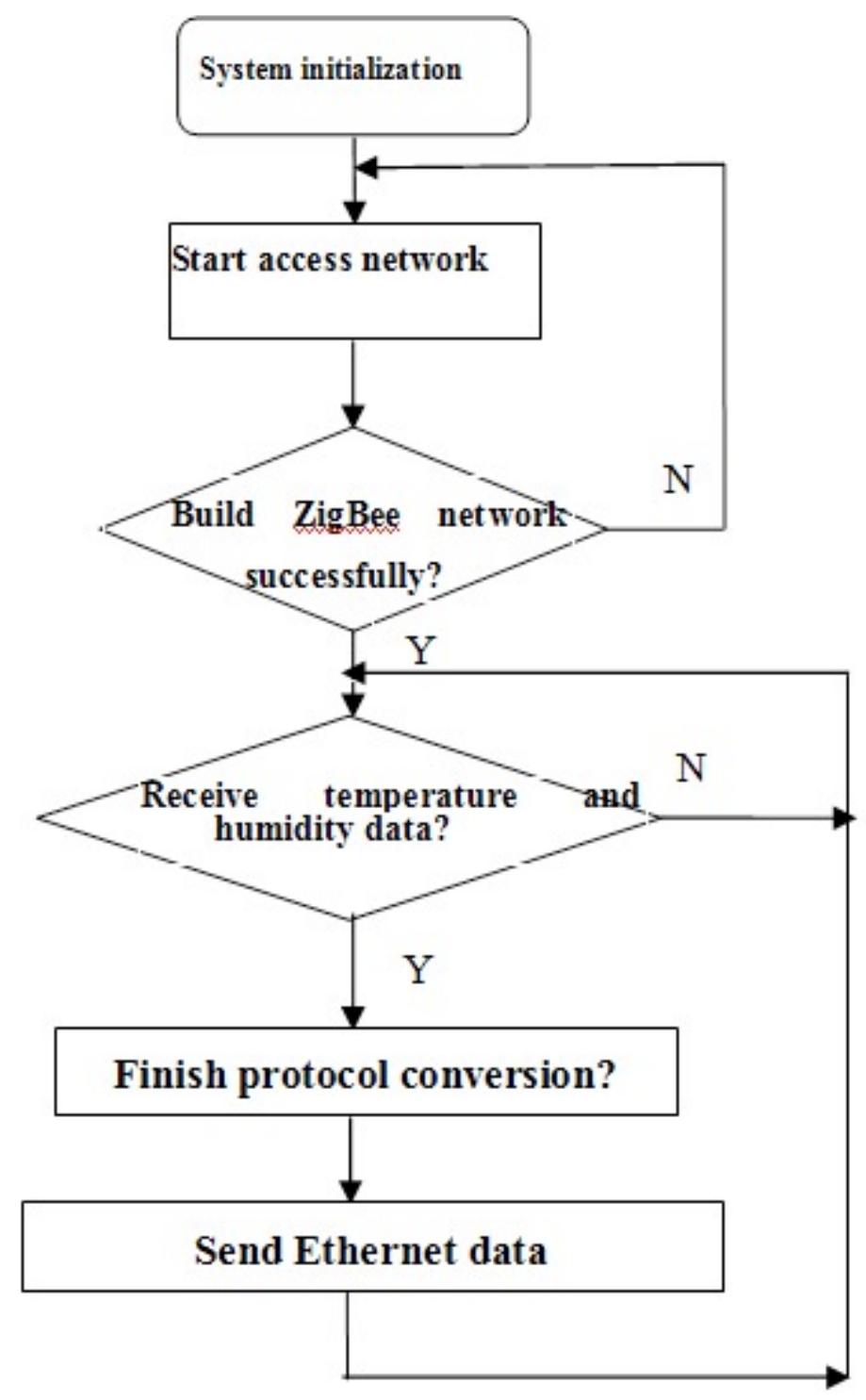

Fig. (2). The algorithm flow chart of coordinator.

Router domain of frame header. To the binding continuous data, application layer of ZigBee chooses the enabled routing way. It means that it makes use of AODVjr to build router finding at first, and then chooses the router whose hop counts are least and become the optimal path; Towards eruptive and discontinuous data, it will choose the way of restraining router finding, which means it will choose Tree routing way when there is not responsive destination node address. Because this router doesn't need to build routing list, it will respond to the transmitting data more quickly [10].

The concrete realization is that Discover Router domain specifies routing data frame header [11]. The domains may have following three values:

Inhibit the route discovery: It uses the routing table already that exists. When the address of the destination node in the routing table is not available, the parameters nwkUseTreeRouting are TRUE. And the network will use a tree router.

Enable the route discovery: If the routing address is in the routing table, it will route in accordance. Otherwise, the router will use AODVjr routing algorithm initial route discovery. If the node does not have the ability of the initial route discovery, it will use the tree router.

Mandatory route discovery: Regardless of whether there is a corresponding routing table, the nodes will mandatorily use AODVjr routing algorithms to initialize routing discovery.

Routing node will be in dormant state after accessesing network successfully, and it will be awaked when receiving data package from sensor node and send data package to coordinator node. This process follows the mode that: dormancy-awaked by receiving data package-send data- dormancy, design flow of the software is shown in Fig. (3).

\section{CONCLUSION}

The hardware design of natural rubber plantation temperature and humidity collecting system in this article are based on Zigbee wireless networking platform. Wireless communication protocols between the various devices use 


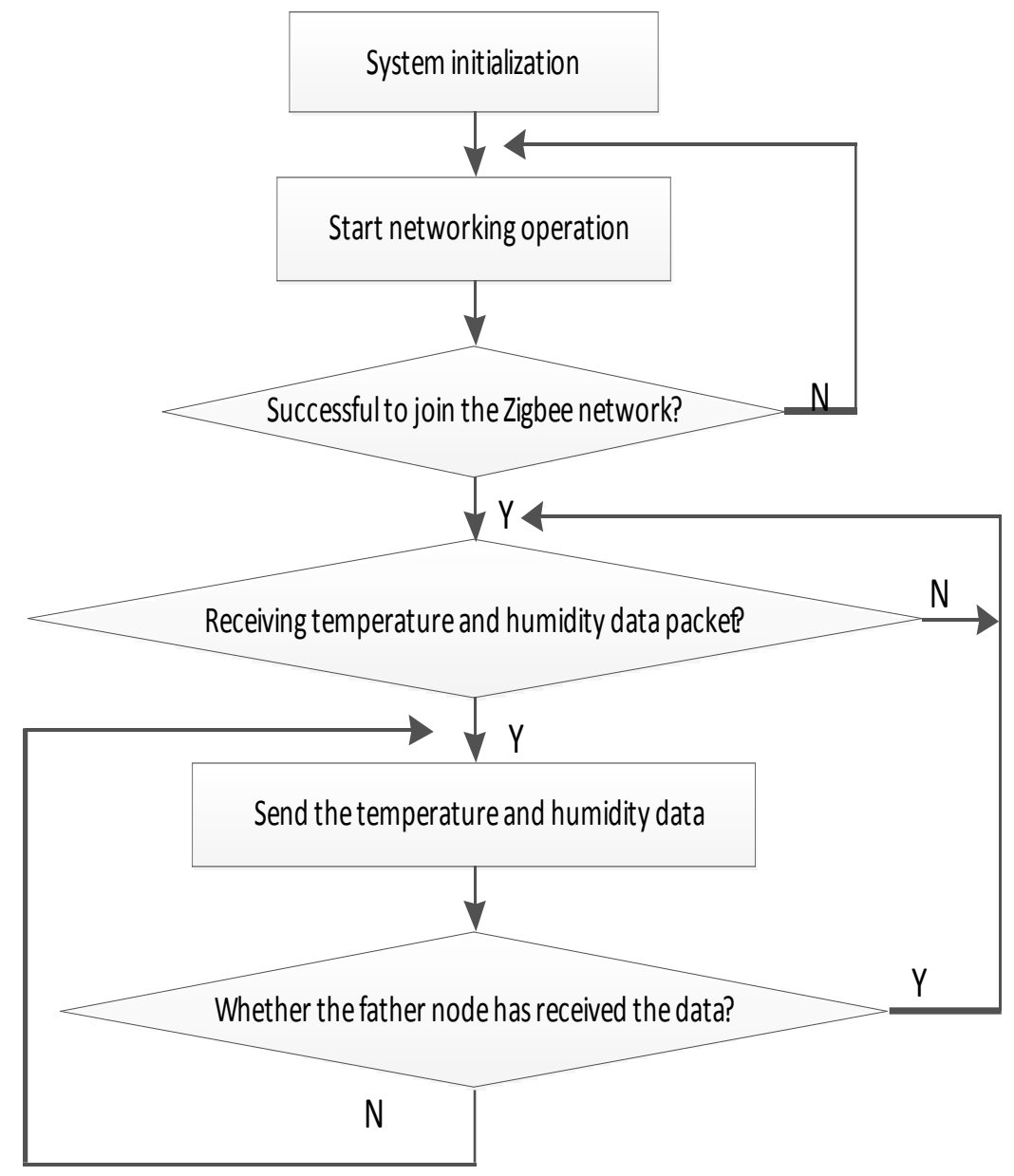

Fig. (3). Routing algorithm flowchart.

the Zigbee protocol. Sensor node is used to collect the realtime data of the temperature and humidity of rubber woods. Then data is transmitted through the wireless network to the router nodes, router nodes according to the routing algorithm select the most suitable communication path in order to collect the value of temperature and humidity from each node. Commands are sent through host PC software to each sensor node to realize the both-side communication and eventually remote monitor the temperature and humidity of the rubber woods. It is a typical example of ad hoc networks using wireless sensing system effectiveness and advantages in the field of natural rubber plantation agricultural equipment monitoring. The system created by the ZigBee network platform and associated online monitoring system will be more flexible in temperature and humidity acquisition system to play its utility.

\section{CONFLICT OF INTEREST}

The authors confirm that this article content has no conflicts of interest.

\section{ACKNOWLEDGEMENTS}

Financial supports from: (1)Acquisition System Design of Temperature and Humidity Based on Wireless Sensor Network(rdzwkfjj006), Hainan Tropical Crops Research of Information Technology Open Fund of Key Laboratory Project;
(2Logistics Development Mode Research of Yangpu Port(hd09xm89), Hainan University 2009 Research Project; (3)Tourism Logistics System Coordination Mechanism and Modeling Analysis Research Based on System Dynamics(CXZZ11_0580), Jiangsu 2011 University Postgraduate Research and Innovation Project.

\section{REFERENCES}

[1] W.U Zhi-xiang, X. Gui-shui, T.A.O Zhong-liang, "The Carbon and Total Nitrogen Characterisitcs of Different Age Rubber Plantation Soil in Danzhou of Hainan", Journal of Ecology and Environmental Sciences, vol. 18, no.4, pp. 1484-1491, 2009.

[2] W.U. Zhi-xiang, X. Gui-shui, and Y. Chuan, "The initial observations of rubber planation ecosystem dry season microclimate characteristics and flux", Journal of Tropical Crops, vol. 12, pp. 20812090, 2010.

[3] LI W-Zhong and D. Chao-yu, ZigBee wireless network technology's introduction and actual combat. Beijing, Beijing University of Aeronautics and Astronautics publishing house, 2007, pp.1-12,

[4] L.U. Zhi-an, The principle and application development of ZigBee network, Beijing: Beijing University of Aeronautics and Astronautics publishing house, 2008, pp. 18-32

[5] Y. Chun-hua,W. Zhang-rui and X. Yu, "The design of wireless network coordinator nodes based on zigbee technology", Electronics Instrumentation Customer, vol. 6, pp.55-60, 2010.

[6] Y. Xia, "Animal robot system based on zigbee wireless sensor network", Beijing, China Science and Technology University 2010

[7] C. Xiangping and F. Li, "Microcomputer Information", vol.25, no. 4, pp. 99-101.

[8] K. Taehong, K. Daeyoung and P. Noseong, "Shortcut tree routing in ZigBee networks", Proceedings of the 2nd International Sympo- 
sium on Wireless Pervasive Computing, SanJuan, Puerto Rico, pp. 32-47, 2007.

[9] I.D Chakems, L. Klein-Berndt and AODVjr, "AODV simplified", ACM SZGMOBIL Mobile Computing and Communications Review, vol. 6, no.3, pp. 100-101, 2002.
[10] L. Juan and G. Youmin, "Railway Computer Application", vol. 18, no.4, pp.34-36, 2009.

[11] W. Guangrong, Z. Jianxiong and X. Xianghua, "Journal of Hangzhou Dianzi" vol. 28, no .4, pp. 49-52, 2008.

Received: August 13, 2013

Revised: August 28, 2013

Accepted: August 28, 2013

(C) Zhi-yang et al.; Licensee Bentham Open.

This is an open access article licensed under the terms of the Creative Commons Attribution Non-Commercial License (http://creativecommons.org/licenses/by-nc/3.0/) which permits unrestricted, non-commercial use, distribution and reproduction in any medium, provided the work is properly cited. 